\title{
A three-pronged strategy to improve trust in biodiversity data produced by citizen science programs
}

\author{
Robert Stevenson $¥$ \\ ‡ University of Massachusetts, Boston, Boston, United States of America
}

Corresponding author: Robert Stevenson (rdstevenson10@gmail.com)

Received: 15 Apr 2018| Published: 17 May 2018

Citation: Stevenson R (2018) A three-pronged strategy to improve trust in biodiversity data produced by citizen science programs. Biodiversity Information Science and Standards 2: e25838.

https://doi.org/10.3897/biss.2.25838

\section{Abstract}

The quality of data produced by citizen science (CS) programs has been called into question by academic scientists, governments, and corporations. Their doubts arise because they perceive CS groups as intruding on the rightful opportunities of standard science and industry organizations, because of a normal skepticism of novel approaches, and because of a lack of understanding of how CS produces data.

I propose a three-pronged strategy to overcome these objections and improve trust in CS data.

1. Develop methods for CS programs to advertise their efforts in data quality control and quality assurance (QCQA). As a first step the PPSR core could incorporate a field that would allow programs to point to webpages that document the QAQC practices of each program. It is my experience that many programs think carefully about data quality, but the CS community currently lacks an established protocol to share this information.

2. Define and implement best practices for generating biodiversity data using different methods. Wiggins et al. 2011 published a list of approaches that can be 
used for QCQA in CS projects but how these approaches should be implemented has not been systematically investigated.

3. Measure and report data quality. If one takes the point of view that citizen science is akin to a new category of scientific instruments, then the ideas of instrument measurement and calibration can be applied CS. Scientists are well aware that any instrument needs to be calibrated before its efficacy can be established. However, because CS is new approach, the specific procedures needed for different kinds of programs are just now being worked out for the first time.

The strategy outlined above faces some specific challenges. Citizen science biodiversity programs must address two important problems that standard scientific entities encounter when sampling and monitoring biodiversity. The first is correctly identifying species. For citizens this can be a problem because they often do not have the training and background of scientist teams. Likewise, it may be difficult for CS projects to manage updating and maintaining the taxonomies of the species being investigated. A second set of challenges is the diverse kinds of biodiversity data collected by CS programs. For instances, Notes from Nature decodes that labels of museum specimens, Snapshot Serengeti identifies species of large mammals from camera trap photographs, iNaturalist collections images of species and then has a crowdsource identification processs, while eBird collects observations of birds that are immediately filtered with computer algorithms for review by the observer and if, subsequently flagged, reviewed by a local expert. Each of these programs likely requires a different set of best practices and methods to measure data quality.

\section{Keywords}

citizen science, biodiversity, data quality, eBird, iNaturalist, Notes from Nature, Snapshot Serengeti, PPSR core

\section{Presenting author}

Robert Stevenson 